\title{
SLC26A3 (DRA) prevents TNF-alpha-induced barrier dysfunction and dextran sulfate sodium-induced acute colitis
}

\author{
Xiangming Ding ${ }^{1} \cdot$ Dongxiao $\mathrm{Li}^{1} \cdot$ Mengke $\mathrm{Li}^{1,2} \cdot \mathrm{Han} \mathrm{Wang}^{1} \cdot \mathrm{Qin} \mathrm{He}^{1} \cdot \mathrm{Yunwu}$ Wang ${ }^{1} \cdot$ Hongbing $\mathrm{Yu}^{3} \cdot$ Dean Tian ${ }^{1} \cdot$ \\ Qin $\mathrm{Yu}^{1}$
}

Received: 17 August 2017 / Revised: 8 November 2017 / Accepted: 15 November 2017

(c) United States \& Canadian Academy of Pathology 2018

\begin{abstract}
SLC26A3 encodes a $\mathrm{Cl}^{-} / \mathrm{HCO}_{3}{ }^{-}$ion transporter that is also known as downregulated in adenoma (DRA) and is involved in $\mathrm{HCO}_{3}{ }^{-}$/mucus formation. The role of DRA in the epithelial barrier has not been previously established. In this study, we investigated the in vivo and in vitro mechanisms of DRA in the colon epithelial barrier. Immunofluorescence (IF) and coimmunoprecipitation (co-IP) studies reveal that DRA binds directly to tight junction (TJ) proteins and affects the expression of TJ proteins in polarized Caco-2BBe cells. Similarly, DRA colocalizes with ZO-1 in the intestinal epithelium. Knockdown or overexpression of DRA leads to alterations in TJ proteins and epithelial permeability. In addition, TNF- $\alpha$ treatment downregulates DRA by activating NF-кB and subsequently affecting intestinal epithelial barrier integrity. Furthermore, overexpression of DRA partly reverses the TNF- $\alpha$-induced damage by stabilizing TJ proteins. Neutralization of TNF- $\alpha$ in dextran sulfate sodium (DSS)-induced colitis mice demonstrates improved the outcomes, and the therapeutic effect of the TNF- $\alpha$ neutralizing mAb is mediated in part by the preservation of DRA expression. These data suggest that DRA may be one of the therapeutic targets of TNF- $\alpha$. Moreover, DRA delivered by adenovirus vector significantly prevents the exacerbation of colitis and improves epithelial barrier function by promoting the recovery of TJ proteins in DSS-treated mice. In conclusion, DRA plays a role in protecting the epithelial barrier and may be a therapeutic target in gut homeostasis.
\end{abstract}

\section{Introduction}

Inflammatory bowel disease (IBD) is a non-specific intestinal inflammatory disease with increasing incidence in recent years [1]. Indeed, a growing body of evidence

Xiangming Ding and Dongxiao Li contributed equally to this work.

Electronic supplementary material The online version of this article (https://doi.org/10.1038/s41374-017-0005-4) contains supplementary material, which is available to authorized users.

$\triangle$ Dean Tian

datian@tjh.tjmu.edu.cn

$\triangle$ Qin Yu

yuqin@tjh.tjmu.edu.cn

1 Department of Gastroenterology, Tongji Hospital, Huazhong University of Science and Technology, Hubei Sheng, China

2 Department of Gastroenterology, Zhoushan Hospital, Zhoushan, China

3 Department of Pediatrics, BC Children's Hospital and the University of British Columbia, Vancouver, BC, Canada indicates that IBD is associated with or may be secondary to defects in the gut barrier [2]. The intestinal epithelial barrier is composed of a layer of columnar cells that separate internal tissues from the luminal content. The paracellular pathway between cells are sealed by tight junctions (TJs) and adherens junctions (AJs), which modulate the integrity and permeability of epithelial monolayers [3, 4]. TJ disruption results in increased mucosal permeability, which allows bacteria and toxins to pass from the lumen into the submucosa, triggering inflammation [5]. TJ disruption is considered as a vital event in the pathogenesis of intestinal inflammation [6, 7], highlighting the importance of exploring TJ protein dynamics.

DRA (downregulated in adenoma) is a $\mathrm{Cl}^{-} / \mathrm{HCO}_{3}{ }^{-}$ exchanger encoded by the gene $S L C 26 A 3$ that contributes to intestinal fluid absorption and enterocyte acid/base balance $[8,9]$. Attention has largely focused on its ion exchange properties. DRA anion exchange activity and expression are both significantly decreased in IBD [10] and related animal models [11]. Recent work suggested that the loss of DRA is associated with decreased mucosal $\mathrm{HCO}_{3}{ }^{-}$secretion, potentially due to inflammation-induced changes in the 
crypt and villous architecture or to the effect of proinflammatory cytokines on epithelial ion transporters $[12,13]$. We previously identified that DRA deficiency is associated with the absence of a firmly adherent mucus layer and with $\mathrm{HCO}_{3}{ }^{-}$/mucus barrier impairment in mice. This change in mucus layer renders $S L C 26 \mathrm{~A}_{3}{ }^{-1-}$ mice susceptible to dextran sulfate sodium (DSS)-induced colitis [14]. DRA was also found to be involved in tumor suppression by maintaining gastric mucosal integrity [15]. DRA regulation of the intestinal epithelial barrier remains to be elucidated.

Previous work in TNF- $\alpha$-overexpressing mice $\left(\mathrm{TNF}^{+/ \triangle \mathrm{ARE}}\right.$ mice) demonstrated that $\mathrm{TJ}$-associated proteins are altered, and DRA expression in the colon was effected [16]. These findings led us to hypothesize that TNF- $\alpha$-induced TJ disruption occurs in part through effects on DRA expression, ultimately exacerbating colon inflammation. To test this hypothesis, we examined DRA localization within TJs in a Caco-2BBe cell monolayer. In addition, mice with DSS-induced colitis were infected with adenovirus encoding DRA and treated with a TNF- $\alpha$ monoclonal antibody (mAb). Epithelial permeability, TJ protein expression, colitis severity, and histological inflammation were then studied in these mice. On the basis of these studies, we discuss the mechanism by which TNF$\alpha$ regulates DRA to further influence the intestinal epithelial barrier.

\section{Materials and methods}

\section{Ethics statement}

All animal work was performed in accordance with the National Institutes of Health Guide for the Care and Use of Laboratory Animals. The protocol was approved by the Committee on the Ethics of Animal Experiments of Tongji Medical College, and the study was monitored by the Department of Experimental Animals of Tongji Medical College.

\section{Antibodies}

The following antibodies were used in this study: DRA antibody (sc-34942, Santa Cruz Biotechnology, Dallas, TX, USA) for immunofluorescence (IF) and co-immunoprecipitation (coIP); ZO-1 antibody (GTX108613, GeneTex, Irvine, CA, USA) for IF and co-IP; occludin antibody (71-1500, Thermo Fisher Scientific, Waltham, MA, USA); $\beta$-actin antibody (TDY051, Beijing TDY Biotech, Beijing, China); DRA antibody (ab83545) for western blot, ZO-1 antibody (ab190085) for western blot, claudin 1 (ab180158), claudin 2 (ab53032) and claudin 5 antibody (ab15106) were purchased from Abcam (Shanghai, China).

\section{Cell culture and treatment}

Caco-2BBe cells were kindly provided by Prof. U. Seidler (Hannover Medical School, Germany) and cultured as previously described [17]. The cells were stable for up to 25 passages (cells were not tested for longer time periods), and all the experiments were performed within less than 25 passages. The DRA-knockdown (KD) cell line was produced using a short hairpin RNA (shRNA) lentiviral vector plasmid (hU6-MCS-CMV-Puromycin GV112, Genechem, Shanghai, China). Recombinant lentivirus overexpressing DRA (pLVX-DRA) was constructed using a lentiviral vector system (Ubi-MCS-3FLAG-SV40-puromycin GV341, Genechem, Shanghai, China). Cells were transfected at $80-90 \%$ confluence for $12 \mathrm{~h}$, and stably transfected cells were selected with puromycin. To simulate the colonic epithelial cells in vivo, Caco-2BBe cells were cultured for at least 1 week under a polarized state. To determine whether NF-кB is involved in regulating DRA expression, Caco-2BBe cells were pretreated with $30 \mu \mathrm{M}$ BAY-11-7082 (Med Chem Express, USA) for $30 \mathrm{~min}$ and then exposed to TNF- $\alpha$ $(100 \mathrm{ng} / \mathrm{mL})$ for $6 \mathrm{~h}$.

\section{Immunofluorescence}

Cells were fixed with $4 \%$ paraformaldehyde at room temperature for $15 \mathrm{~min}$ and then permeabilized with $0.3 \%$ Triton X-100 in PBS for $10 \mathrm{~min}$ at room temperature. For paraffin-embedded tissue sections, routine dewaxing, rehydration and antigen retrieval were performed before permeabilization. After permeabilization, cells or tissues were blocked with $1 \%$ BSA in PBS for 30 min, incubated overnight with primary antibodies diluted in $1 \%$ BSA in PBS at $4{ }^{\circ} \mathrm{C}$, and washed 3 times with PBS. Then, the cells or tissues were incubated with the appropriate secondary antibody. Images were acquired at room temperature using an Olympus laser scanning confocal microscope equipped with a Plan Apo $60 \times 1.40$ NA oil immersion lens and controlled by FV1000 Viewer software (Olympus, Tokyo, Japan) and were further processed using Imaris (Bitplane, Concord, MA, USA).

\section{Co-immunoprecipitation}

For co-IP assays, Caco-2BBe cells were lysed on ice with $1 \% \mathrm{NP}-40$ lysis buffer for $30 \mathrm{~min}$. After centrifugation, supernatants were incubated overnight at $4{ }^{\circ} \mathrm{C}$ with protein G-conjugated Sepharose beads (Santa Cruz Biotechnology, Dallas, TX, USA) and the appropriate antibodies. The beads were then washed four times with lysis buffer, and the precipitates were eluted with sample buffer, separated by SDS/PAGE, and analyzed by immunoblotting. 
A

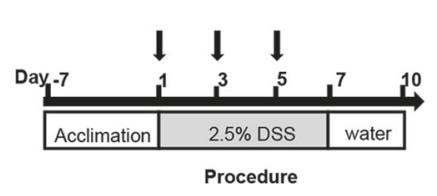

B

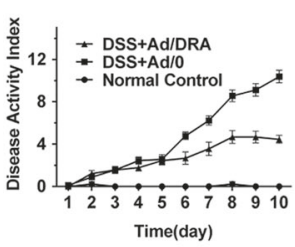

C

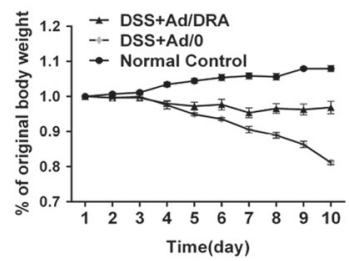

D

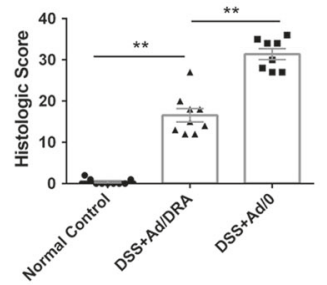

$\mathrm{E}$

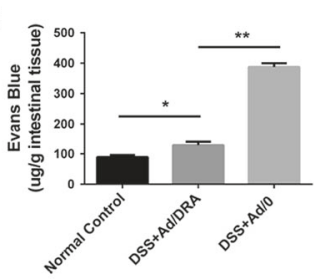

$\mathbf{F}$

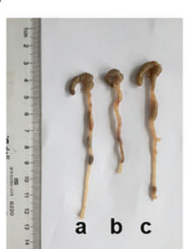

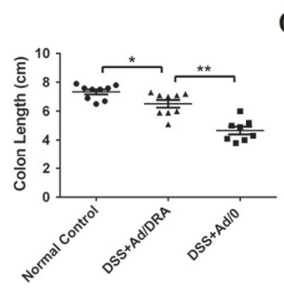

Normal Control

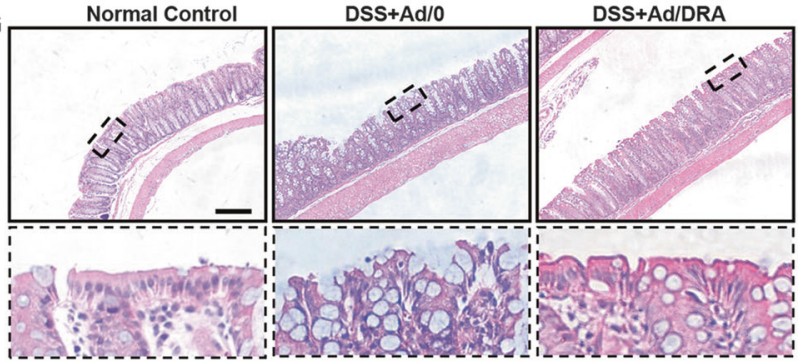

H

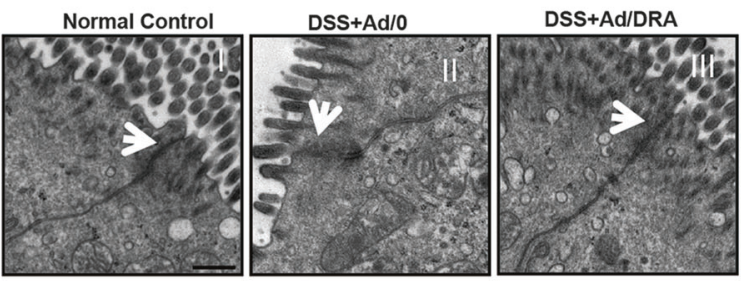

I

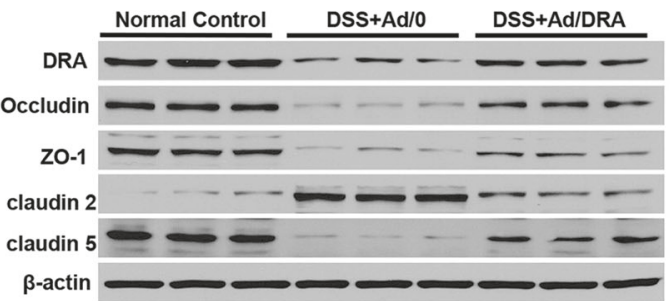

$85 \mathrm{kDa}$

$53 \mathrm{kDa}$

195 kDa

$25 \mathrm{kDa}$

$24 \mathrm{kDa}$

$43 \mathrm{kDa}$
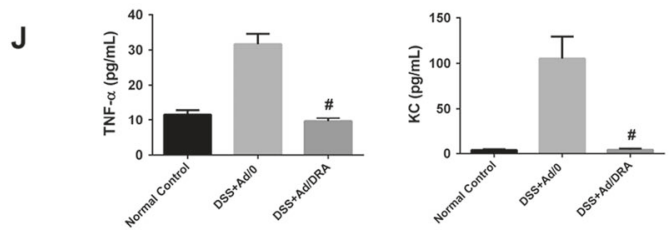

Fig. 1 Delivery of Ad-DRA attenuates DSS-induced colitis with improved epithelial barrier function. a Overview of the procedure. Mice in the normal control group were allowed free access to tap water, and the other two groups were treated orally with DSS water $(2.5 \%)$ for 7 days and then tap water for 3 days, as described in the methods. The data for day 1 were collected before oral DSS administration. The time of recombinant purified adenovirus intervention is indicated by the black arrow. b Disease activity index over 10 days. $P$ $<0.05$ from day 5 to day $10, \mathrm{Ad} / 0$ control vs. Ad/DRA. Statistical significance was determined using the Holm-Sidak multiple comparison test with alpha $=5.000 \%$. c Body weight measurements over 10 days are presented as the $\%$ of original body weight $(P<0.05$ from day 6 to day 10). d Histologic scores of mice in different treatment groups. $N=8$ in the $\mathrm{Ad} / 0$ treatment group (one mouse died on day $10) ; N=9$ in the other two groups $(* * P<0.01)$. e Intestinal permeability measured by Evans blue assay. The data are presented as the mean $\pm \mathrm{SE}$, with 6 mice per experimental group (chosen based on having a representative histologic score, $89.47 \pm 5.98$ vs. $128.70 \pm$

\section{Transepithelial electrical resistance (TEER)}

Caco-2BBe cells were cultured on 0.4- $\mu \mathrm{m}$ 12-well hanging cell culture inserts (Corning, NY, USA) for 21 days. A total of $5 \times 10^{5}$ cells were seeded in $0.5 \mathrm{~mL}$ of medium in the inserts, and the basolateral chamber was filled with $1.5 \mathrm{~mL}$

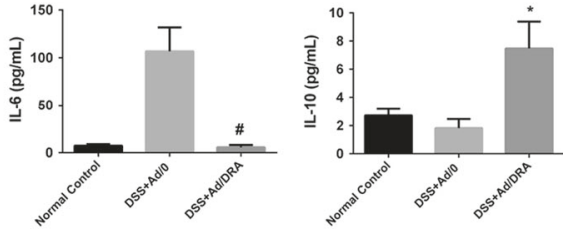

$10.96 \mu \mathrm{g} / \mathrm{g}, * P<0.05 ; 128.70 \pm 10.96$ vs. $387.50 \pm 12.09 \mu \mathrm{g} / \mathrm{g}, * * P<$ 0.01). f Colon length in each group. "a" indicates the normal group, "b" indicates the DSS + Ad/0 group, and "c" indicates the DSS + Ad/DRA group $(7.33 \pm 0.17$ vs. $6.51 \pm 0.26 \mathrm{~cm}, * P<0.05 ; 6.51 \pm 0.26$ vs. 4.66 $\pm 0.26 \mathrm{~cm}, * * P<0.01)$. The remaining colon samples are shown in the Supplemental Information. $\mathbf{g}$ Representative histological images of the distal colon (scale bar $=200 \mu \mathrm{m}$ ). $\mathbf{h}$ Ultrastructure micrographs of TJs in the mouse colon. Scale bar $=0.5 \mu \mathrm{m}$. i. TJs are intact in normal mice. II. The arrow indicates that the TJ architecture was altered, with significantly shorter and more sparse microvilli on the free surface of epithelial cells. III. TJs showed minor disruption and separation with regular microvilli in the Ad-DRA-treated group. i Representative western blots of DRA, ZO-1, occludin, claudin 2 and claudin 5 in isolated colonic tissues. $\mathbf{j}$ Cytokine expression in serum at day 10 . Serum cytokine levels were analyzed by flow cytometry with a CBA inflammatory kit, and the data are presented as the mean \pm SE of 6 mice (chosen based on having a representative histologic score). $* P<$ 0.05 , one-way ANOVA. \# $P<0.05$ compared with the Ad/0 group

of medium. The medium was changed every 2 days for the first week and then daily until analysis. Caco-2BBe cells were pretreated with or without $100 \mathrm{ng} / \mathrm{mL}$ TNF- $\alpha$ (Pepro Tech, Rocky Hill, NJ, USA) for $24 \mathrm{~h}$ before transepithelial electrical resistance (TEER) measurement. Calcium- and magnesium-free Hanks Balanced Salt Solution (HyClone, 
Table 1 Scoring of disease activity index (DAI)

\begin{tabular}{llll}
\hline Score & Weight loss $(\%)$ & Stool consistency $^{\mathrm{a}}$ & Occult/gross bleeding $^{\text {Ond }}$ \\
\hline 0 & None & Normal & Normal \\
1 & $1-5$ & & \\
2 & $5-10$ & Loose stools & Hemoccult + \\
3 & $10-15$ & & \\
4 & $>15$ & Diarrhoea & Gross bleeding \\
\hline
\end{tabular}

The DAI value is calculated as the sum of scores of \% weight loss, stool consistency and blood in feces

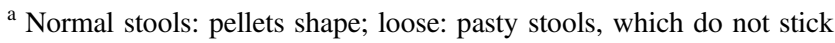
to the anus; diarrhoea: liquid stools that stick to the anus

Logan, Utah, USA) was used to wash the cells. TEER values were detected at $37^{\circ} \mathrm{C}$ using an epithelial vol/ohm meter (World Precision Instruments Inc., Sarasota, FL, USA). Final values were obtained by subtracting the blank value, and the results are expressed in $\Omega^{*} \mathrm{~cm}^{2}$. All TEER values were obtained from three independent trials performed in triplicate.

\section{Animals and experimental protocol}

Male C57BL/6 mice (6-8 weeks old) were purchased from the Department of Experimental Animals of Tongji Medical College (Wuhan, China). The use of laboratory animals was approved by the China Medical University Institutional Animal Care and Use Committee. All C57BL/6 mice were housed together for 1 week prior to the study, after which they were randomly divided into 3 groups of 9 mice each. Mice in the DSS control and experiment groups were given $2.5 \%$ DSS solution [2.5 g DSS powder (MW 36,000-50,000; MP Biomedicals, Solon, OH, USA) in 100 $\mathrm{mL}$ of drinking water] for 7 days, followed by normal water for another 3 days, based on methods described in a previous study [18].

Gene transfer was achieved via intracolonic administration of purified mouse type 5 adenovirus harboring the DRA gene from Hanbio Biotechnology Co., Ltd. (Shanghai, China). DRA delivery was achieved by intracolonic administration of $1 \times 10^{9}$ plaque-forming units of DRA (Ad/DRA) or null (Ad/0) type 5 adenovirus on days 1,3 , and 5 (Fig. 1a). The route and time course of adenovirus administration were based on a previous study [19]. For the second part of the animal experiment, InVivoMAb antimouse TNF $\alpha$ (Bio X Cell, West Lebanon, NH, USA) was given to the experimental group via intraperitoneal injection, while the DSS control group received mouse IgG1 isotype under the same conditions as a negative control. The dosage was $0.5 \mathrm{mg}$ per mouse on days 4 and 6 .

To evaluate colitis severity, we assessed the disease activity index (DAI) score daily, as described by others [20, 21]. The histologic score is the sum of the inflammation
Table 2 Histologic scoring

\begin{tabular}{|c|c|c|}
\hline Feature scored & Score & Description \\
\hline \multirow[t]{4}{*}{ Severity of inflammation } & 0 & None \\
\hline & 1 & Mild \\
\hline & 2 & Moderate \\
\hline & 3 & Severe \\
\hline \multirow[t]{4}{*}{ Extent of inflammation } & 0 & None \\
\hline & 1 & Mucosa \\
\hline & 2 & Mucosa and submucosa \\
\hline & 3 & Transmural \\
\hline \multirow[t]{5}{*}{ Crypt damage } & 0 & None \\
\hline & 1 & Basal $1 / 3$ damaged \\
\hline & 2 & Basal $2 / 3$ damaged \\
\hline & 3 & $\begin{array}{l}\text { Crypts lost; surface epithelium } \\
\text { present }\end{array}$ \\
\hline & 4 & $\begin{array}{l}\text { Crypts lost and surface epithelium } \\
\text { lost }\end{array}$ \\
\hline \multirow[t]{5}{*}{ Percentage involvement } & 0 & $0 \%$ \\
\hline & 1 & $1-25 \%$ \\
\hline & 2 & $26-50 \%$ \\
\hline & 3 & $51-75 \%$ \\
\hline & 4 & $76-100 \%$ \\
\hline
\end{tabular}

Histologic scores were calculated by multiplying the score for the first three parameters by their percentage involvement, giving a maximum score of 40

severity, inflammation extent, and crypt damage divided by the percentage involvement [20]. The specific methods used to calculate the DAI and histologic score are shown in Tables 1 and 2.

\section{Transmission electron microscopy (TEM)}

After isolation from the mice, distal colon tissues were lavaged with $0.9 \%$ saline and then fixed with glutaraldehyde. The remaining tissue preparation steps were performed by the electron microscopy center of Wuhan University. Images were acquired using a transmission electron microscope (Hitachi Model HT7700, Tokyo, Japan).

\section{Assessment of intestinal permeability}

Epithelial permeability was assayed using the Evans blue (EB; Sigma-Aldrich Corp, St. Louis, MO, USA) method, as described previously [22-24]. Because EB may influence the detection of cytokines in serum, the distal small intestine of mice in each group was incised to investigate intestinal permeability to EB. The small intestinal epithelium is the first to be affected in DSS-induced colitis [23, 25] and is therefore appropriate for permeability measurements. The $\mathrm{EB}$ results are expressed in $\mu \mathrm{g} \mathrm{EB} / \mathrm{g}$ intestinal tissue. 


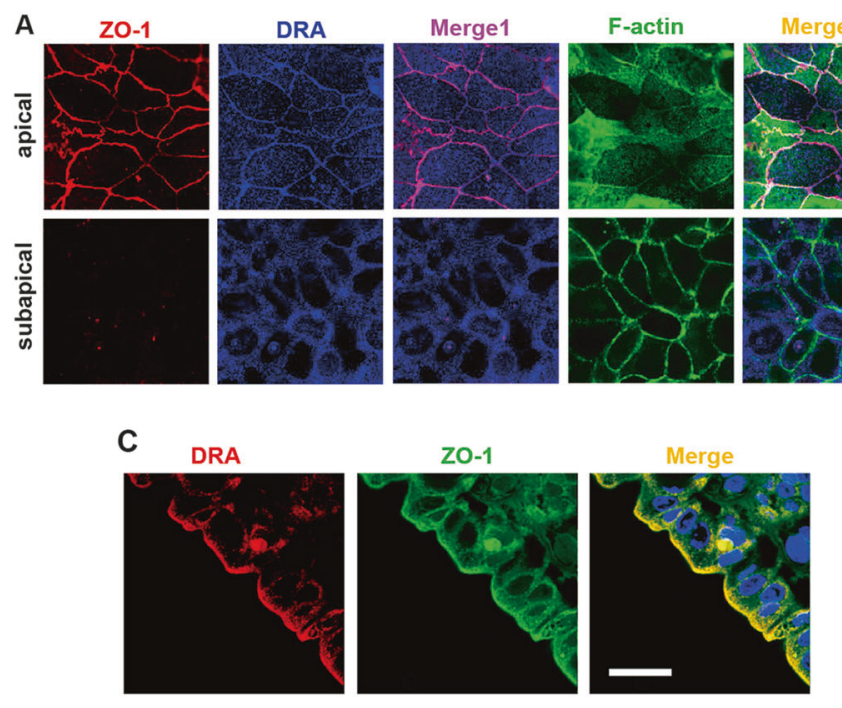

Fig. 2 Identification of DRA as a ZO-1 binding partner along with Factin in TJs. a Triple staining for DRA (blue), ZO-1 (red) and F-actin (green) in polarized Caco-2BBe cells. Single confocal planes at the apical (top) and subapical (bottom) region are shown. Scale bar $=15$ $\mu \mathrm{m}$. b Double staining for DRA (blue) and F-actin (green) in polarized Caco-2BBe cells. Single confocal planes at the basal region (top) and $X Z$ section (bottom) are shown. Scale bar $=15 \mu \mathrm{m}$. c Double staining

\section{Cytokine analysis}

At day 10, blood was collected through the eye, and the mice were killed. Mouse cytometric bead array (CBA) inflammatory kits (BD Biosciences, San Diego, CA, USA) were used to detect the serum levels of TNF- $\alpha$, interleukin (IL)-10, IL-6, and keratinocyte-derived chemokine (KC), according to the manufacturer's specifications. The samples were analyzed by flow cytometry using Flow Cytometric Analysis Program (FCAP) Array software (BD Biosciences).

\section{Luciferase reporter analysis}

Plasmids used for functional analysis of DRA promoter activity were constructed in the $\mathrm{pGL}_{3}{ }^{-}$basic vector carrying a luciferase reporter gene. Mutation of the critical potential binding site of the DRA promoter was accomplished by site-directed mutagenesis by Hanbio Biology Company (Shanghai, China) and confirmed by sequencing. Caco$2 \mathrm{BBe}$ cells were seeded in 24-well plates and transfected at $50-70 \%$ confluence. Briefly, $0.8 \mu \mathrm{g}$ of wild-type (WT) or mutant (MUT) pGL3-DRA and $50 \mathrm{ng}$ of pRL-TK plasmid were mixed with the diluted reagent containing Lipofectamine 3000 (Thermo Fisher Scientific, Waltham, MA, USA) and then added to the cells according to the manufacturer's instructions. The medium was then replaced, and the cells were stimulated with $100 \mathrm{ng} / \mathrm{mL}$ TNF- $\alpha$ for $6 \mathrm{~h}$ prior to processing for the luciferase assay (Promega, Madison, WI, USA). For co-transfection experiments, the transfections

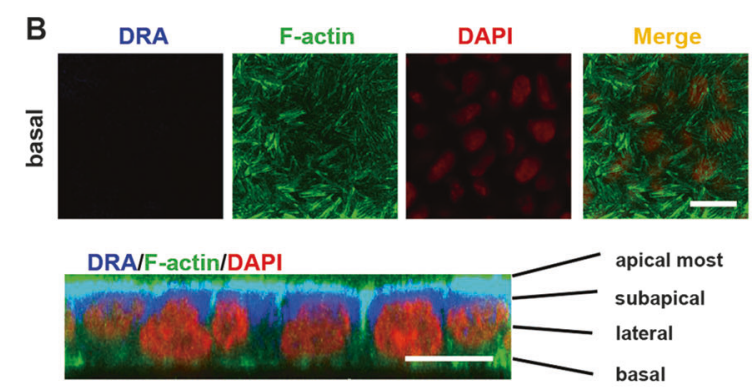

D

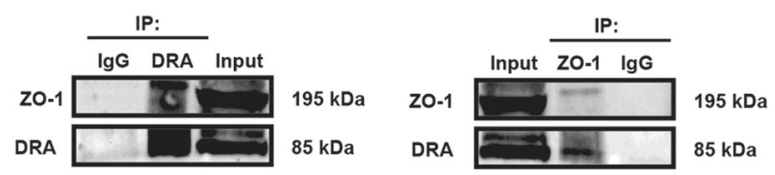

for DRA (red) and ZO-1 (green) in the normal mouse distal colon. Scale bar $=15 \mu \mathrm{m}$. d Endogenous IP assays of Caco-2BBe cell lysates showing that DRA coprecipitated with ZO-1 (left panel). Reciprocal co-IP confirmed that DRA was present in $\mathrm{ZO}-1$ immunoprecipitates (right panel). IgG was used as a negative control

included an additional $0.5-1 \mu \mathrm{g}$ of NF- $\mathrm{KB}$ expression plasmid (Vigene Biosciences, Rockville, MD, USA). DRA promoter region analyses were performed using the NCBI database. Putative binding sites for NF-кB were predicted using the JASPAR CORE database and PROMO (TRANSFAC 8.3).

\section{Statistical analysis}

The data are presented as the mean \pm SE. One-way analysis of variance or Student's $t$ tests were performed to assess the significance of differences among treatment groups. $P<0.05$ indicated statistical significance.

\section{Results}

\section{DRA localizes to TJs in polarized Caco-2BBe cell monolayers}

To explore the physiological functions of DRA in the epithelial barrier, we first examined its subcellular localization in polarized Caco-2BBe cells. Triple IF staining revealed that at the apical region, DRA colocalized with F-actin and the TJ markers ZO-1 along the cell ${ }^{-}$cell borders (Fig. 2a). Intriguingly, DRA signals were also detected at the subapical region where ZO-1 staining was almost absent. Closer inspection showed that granular DRA staining decorated the dense F-actin networks near the cell-cell 

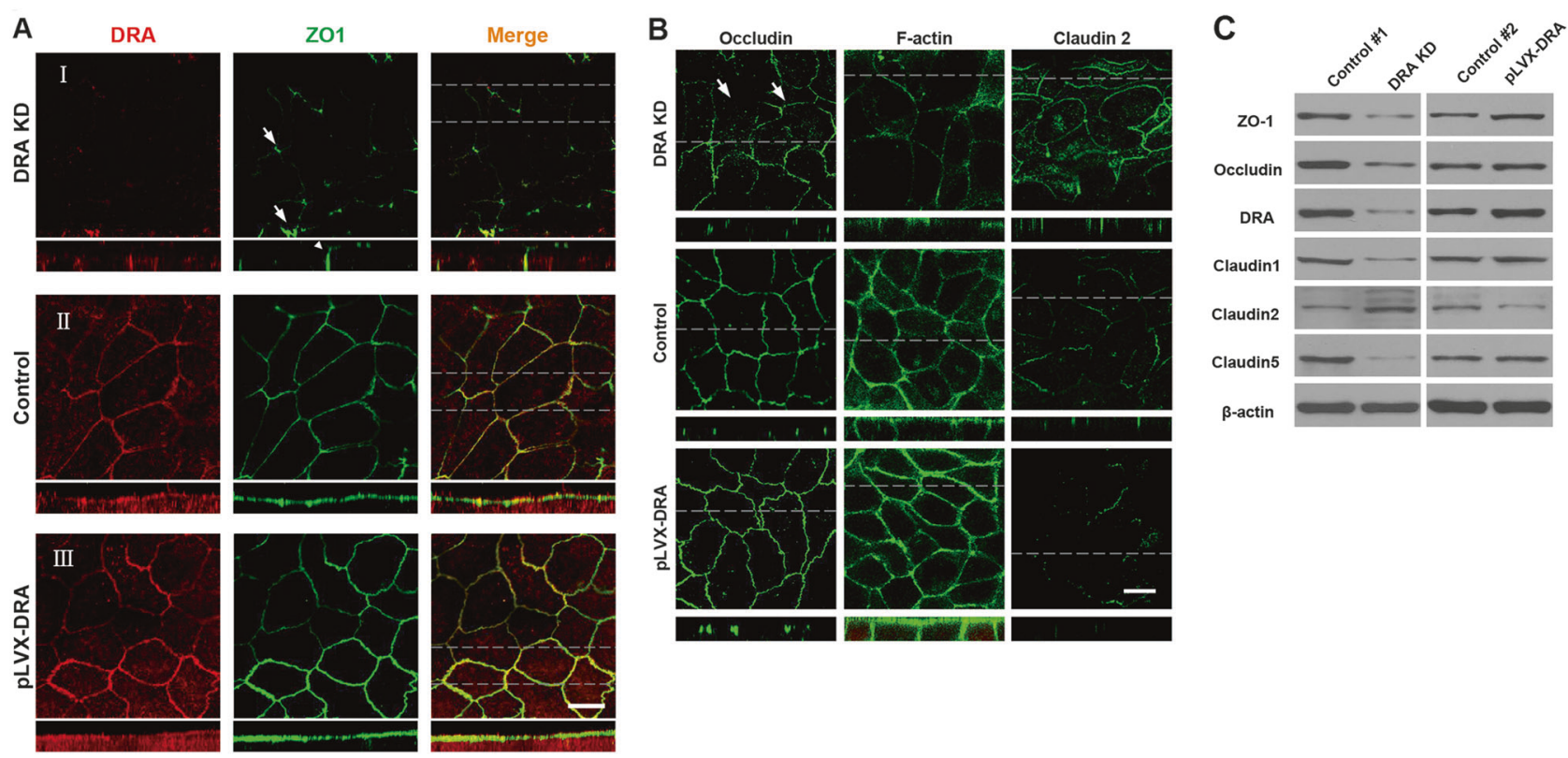

Fig. 3 DRA regulates epithelial barrier integrity in vitro. a Double staining for DRA (red) and ZO-1 (green) in DRA KD (I), control (II) and pLVX-DRA-transfected (III) Caco-2BBe monolayers. The top and corresponding bottom rows show projections of $X Y$ planes and $X Z$ sections, respectively. The images of $X Z$ sections were taken at the position indicated by the two white lines in the $X Y$ sections. Arrows indicate ZO-1 redistribution from bicellular junctions to tricellular tight junctions in DRA KD monolayers. The triangle shows that ZO-1 relocated to the lateral region in DRA KD cells. Scale bar $=15 \mu \mathrm{m}$. b Immunofluorescence staining for occludin, F-actin and claudin 2 in

boundary, but there was relatively less DRA/F-actin colocalization in this region. $X Z$ sections of filter-grown Caco$2 \mathrm{BBe}$ monolayers revealed an apical and lateral distribution of DRA, whereas no DRA staining was detected in the basal region (Fig. 2b). DRA also colocalized with $\mathrm{ZO}-1$ in the normal distal colon (Fig. 2c). Endogenous co-IP assays revealed that DRA was immunoprecipitated by the ZO-1 antibody in Caco-2BBe cells. Similarly, ZO-1 was detected in DRA immunoprecipitates (Fig. 2d). Taken together, the data indicate that DRA is a binding partner of ZO-1 and Factin and localizes to TJs in polarized Caco-2BBe cells, and this localization provides a platform for DRA at the epithelial barrier.

\section{DRA is necessary for maintaining epithelial barrier integrity}

To further characterize the role of DRA in TJs, we first knocked down DRA in Caco-2BBe cells (Fig. 3c). After DRA depletion, there was weaker ZO-1 staining along the bicellular TJs, and ZO-1 was redistributed to tricellular TJs, where residual DRA was present (arrow, Fig. 3a, i). Unexpectedly, $X Z$ stacks showed that ZO-1 was almost absent from the apical region but was relocated to the lateral
DRA KD, DRA-overexpressing and control Caco-2BBe cells. The top and corresponding bottom rows show projections of $X Y$ planes and $X Z$ sections, respectively. The images of $X Z$ sections were taken at the position indicated by the white line in $X Y$ planes. Arrows indicate DRA KD-induced occludin internalization in Caco-2BBe cells. Scale bar: $15 \mu \mathrm{m}$. $\mathbf{c}$ Immunoblotting for DRA and TJ components, including ZO-1, occludin, claudin 1 , claudin 2 and claudin 5 , in DRA KD and DRA-overexpressing Caco-2BBe cells, as well as their respective controls

region in DRA KD cells (triangle, Fig. 3a, i). Moreover, immunoblotting revealed a reduction in $\mathrm{ZO}-1$ protein expression in DRA KD cells (Fig. 3c). In addition, IF staining revealed that exogenously expressed DRA selectively colocalized with ZO-1 along cell-cell borders in the apical region but showed no correlation with ZO-1 in the basal region (Fig. 3a, III). Furthermore, both immunostaining and immunoblotting revealed higher $\mathrm{ZO}-1$ expression in DRA-overexpressing Caco-2BBe cells.

Next, we investigated the effects of DRA on other TJ components (Fig. 3b). Intriguingly, F-actin attachment was apparently weakened, and F-actin no longer accumulated at the apical and lateral regions in DRA KD cells. In contrast, the F-actin fluorescence signal intensity was stronger in pLVX-DRA-transfected Caco-2BBe cells. Occludin staining was weaker and discontinuous after DRA depletion but concentrated at cell-cell borders in DRA-overexpressing Caco-2BBe cells. Moreover, both $X Y$ and $X Z$ sections showed higher occludin internalization in DRA KD cells than in control or DRA-overexpressing cells (arrow, Fig. 3b). Claudin 2 is known as a "leaking" claudin; its upregulation contributes to a leaky gut. Increased claudin 2 expression has been found in both UC and CD patients [26]. Compared with other TJ proteins, claudin 2 showed the 

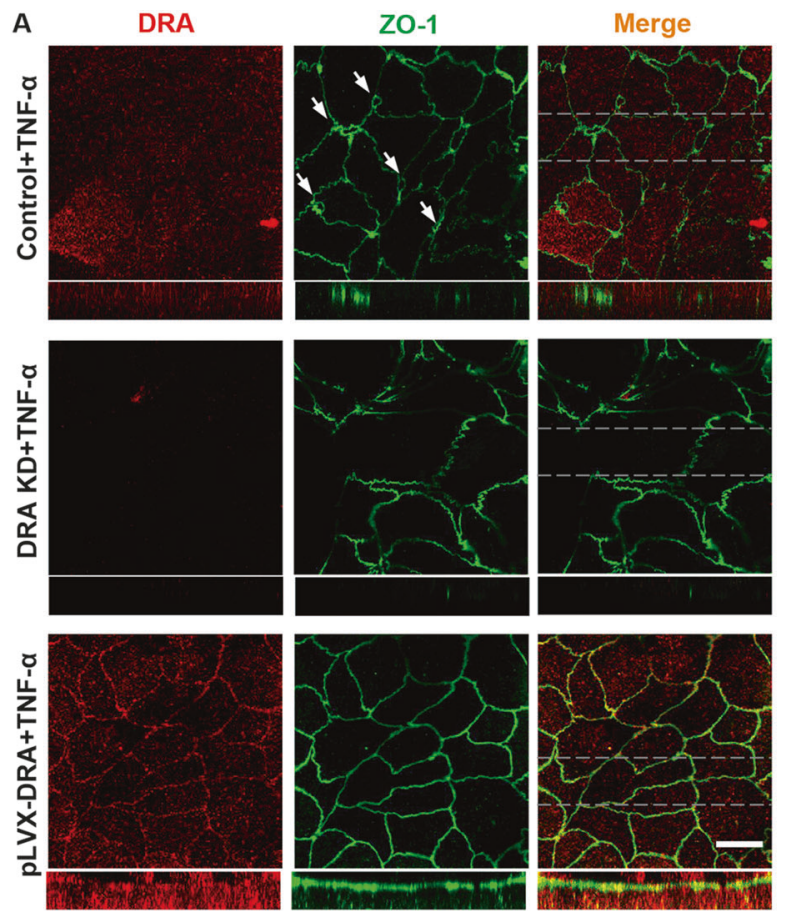

B
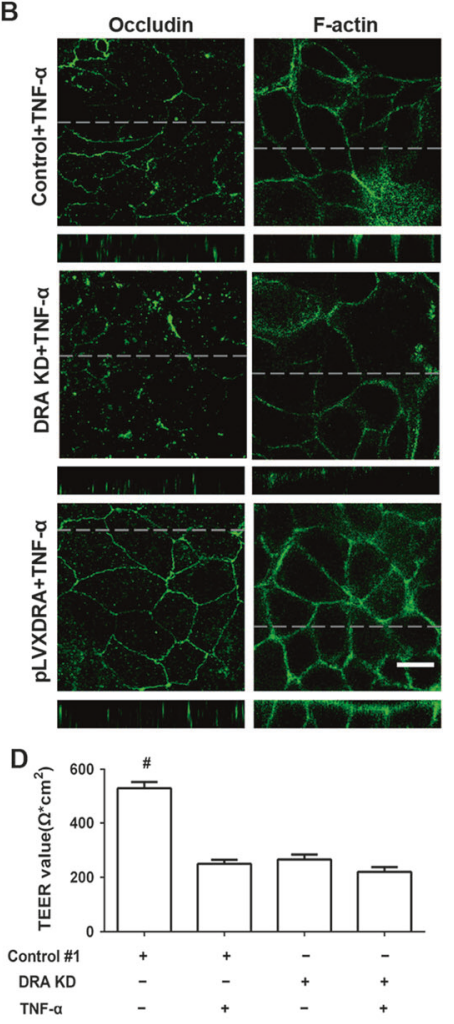
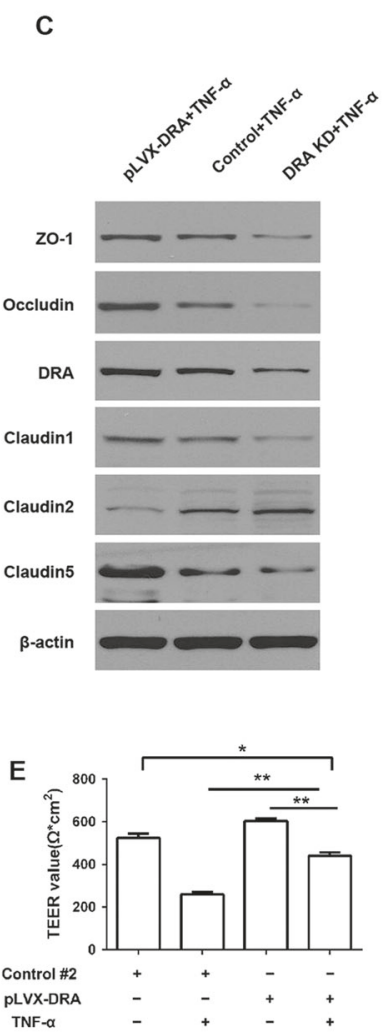

Fig. 4 DRA overexpression strengthens the epithelial barrier, enhances the resistance to damage by TNF- $\alpha$ and is associated with changes in TEER values. a Double staining for DRA (red) and ZO-1 (green) in DRA KD, DRA-overexpressing and control Caco-2BBe cells treated with $100 \mathrm{ng} / \mathrm{mL} \mathrm{TNF-} \alpha$ for $24 \mathrm{~h}$. The top and corresponding bottom rows show projections of $X Y$ planes and $X Z$ sections, respectively. Arrows show that ZO-1 localization was characterized by a ring-like appearance at points of multiple cellular contacts after TNF- $\alpha$ treatment of control Caco-2BBe cells. Scale bar $=15 \mu \mathrm{m}$. b Immunofluorescence staining for occludin and F-actin in DRA KD, DRAoverexpressing and control Caco-2BBe cells. The top and corresponding bottom rows show projections of $X Y$ planes and $X Z$ sections, respectively. The images of $X Z$ sections were taken at the position indicated by a white line in the $X Y$ planes. Scale bar $=15 \mu \mathrm{m}$. c

opposite localization pattern in DRA KD and DRAoverexpressing Caco-2BBe cells. Alterations in TJ proteins were also evaluated by immunoblotting (Fig. 3c). The expression levels of occludin, claudin 1 and claudin 5 were significantly lower in DRA KD cells, whereas claudin 2 expression was higher. In response to DRA overexpression, ZO-1 and occludin levels were increased, and claudin 2 expression decreased; there were no significant changes in claudin 1 or 5 levels.

We therefore measured TEER in DRA KD, DRAoverexpressing and normal polarized Caco-2BBe cells. Compared with normal cells, DRA-depleted cells showed much lower TEER values $(530.3 \pm 22.75$ vs. $265.7 \pm 18.02$ $\Omega * \mathrm{~cm}^{2}, P<0.01$, Fig. 4d). Consistent with these results, the TEER value in DRA-overexpressing cells was higher than

Immunoblotting for DRA and TJ components, including ZO-1, occludin, claudin 1 , claudin 2 , and claudin 5 , in DRA KD, DRAoverexpressing and control Caco-2BBe cells after treatment with TNF$\alpha$ for $24 \mathrm{~h}$. d, e TEER values in DRA KD, DRA-overexpressing and control cells in the absence or presence of TNF- $\alpha$. d Knocking down DRA in Caco-2BBe cells significantly decreased the TEER value compared with control Caco-2BBe cells. TNF- $\alpha$ treatment resulted in lower TEER values in control shRNA cells but had no further effect on the TEER value in DRA KD cells $(\# P<0.05$ compared with all other groups). e DRA overexpression increased the TEER value compared with the empty vector. In addition, DRA overexpression restored the TNF- $\alpha$-induced decrease in TEER value $(* P=0.0286, * * P<0.01)$

that in control cells $(602.7 \pm 12.73$ vs. $524.3 \pm 19.06$ $\Omega * \mathrm{~cm}^{2}, P<0.01$, Fig. $\left.4 \mathrm{e}\right)$.

\section{DRA overexpression strengthens the epithelial barrier and mitigates TNF- $a$-induced damage}

Previous work showed that DRA expression is downregulated in a TNF- $\alpha$-overexpressing mouse model and that TNF- $\alpha$ can affect the expression of TJ proteins [16]. We have also confirmed that TNF- $\alpha$ significantly decreases DRA expression in a dose-dependent manner over a 24-h treatment period (Unpublished). To determine whether DRA protects the epithelial barrier against TNF- $\alpha$, Caco-2BBe cells were treated with TNF- $\alpha(100 \mathrm{ng} / \mathrm{mL})$ for $24 \mathrm{~h}$. 
Epithelial barrier function was assessed by measuring TEER (Fig. 4e). Upon TNF- $\alpha$ treatment, DRAoverexpressing cells had a higher TEER value than cells harboring the vector control $(440.3 \pm 16.25$ vs. $259.0 \pm$ $\left.10.79 \Omega * \mathrm{~cm}^{2}, P<0.01\right)$. Notably, compared with normal control cells, DRA-overexpressing cells still had a significantly decreased TEER value after TNF- $\alpha$ treatment $(P<0.05)$.

We then performed IF to assess the cellular distribution of DRA and TJ proteins after TNF- $\alpha$ treatment. In control Caco-2BBe cell monolayers, TNF- $\alpha$ treatment $(100 \mathrm{ng} / \mathrm{mL})$ led to an almost complete disappearance of DRA at the cell borders and slightly higher cytosolic DRA levels. Meanwhile, TNF- $\alpha$ induced variability in the ZO-1 profile and resulted in reduced colocalization of ZO-1 and DRA at the apical regions, as shown in the $X Y$ and $X Z$ sections. Although ZO-1 showed a ring-like appearance at points of multiple cellular contacts (arrow, Fig. 4a), the continuity of ZO-1 along cellular borders was not remarkably disrupted following exposure to TNF- $\alpha$ for $24 \mathrm{~h}$. DRA overexpression prevented the TNF- $\alpha$-induced disturbance of ZO-1 localization at cellular junctions and partially recovered ZO-1/ DRA colocalization in the apical region. To further determine whether the effect of TNF- $\alpha$ on ZO-1 localization was dependent on DRA, we treated DRA KD Caco-2BBe cells with TNF- $\alpha$; the outlines of ZO-1 were significantly distorted, and the continuity of $\mathrm{ZO}-1$ expression was significantly interrupted. In addition, TNF- $\alpha$ altered occludin and $\mathrm{F}$-actin localization, similar to the effects on $\mathrm{ZO}-1$, in control and DRA-overexpressing Caco-2BBe cells (Fig. 4b). Notably, occludin staining in DRA KD Caco$2 \mathrm{BBe}$ monolayers was weaker and discontinuous and showed more internalization into cytoplasmic vesicles in response to TNF- $\alpha$ treatment. Moreover, actin bundles were disorganized and disappeared at the lateral regions. In contrast, F-actin remained intact, and occludin was still localized to apical domains in pLVX-DRA-transfected Caco-2BBe cells treated with TNF- $\alpha$.

We further investigated changes in TJ proteins by immunoblotting (Fig. 4c). In association with the reduction in DRA expression, the levels of ZO-1, occludin, claudin 1, and claudin 5 were significantly decreased, whereas claudin 2 expression increased in the DRA KD group after TNF- $\alpha$ treatment. TNF- $\alpha$-treated DRA-overexpressing cells showed elevated DRA, ZO-1, occludin, claudin 1, and claudin 5 levels and decreased claudin 2 levels.

\section{Delivery of Ad-DRA ameliorates DSS-induced experimental colitis in mice and reduces TNF-a levels}

To determine whether DRA has potential to modulate IBD, we evaluated this therapeutic approach in a DSS-induced colitis model. The colitis severity and morphological parameters were measured over 10 days. Ad-DRA-treated mice had significantly lower DAI scores from the 6th day of DSS treatment onward compared with Ad-0 control mice $(P<$ 0.05 , Fig. $1 b)$. Mice treated with Ad-DRA lost significantly less weight (from day 6 onward, $P<0.05$, Fig. 1c) and had a significantly longer colon $(P<0.01$, Fig. 1f) than Ad-0treated controls; colon length is regarded as an additional macroscopic indicator of damage from colitis. On the 10th day after initiating DSS feeding, morphological epithelial damage was observed in the Ad-0 control group and confirmed by blind scoring of the colon. In the Ad- 0 controls, colonic histopathology showed a hyperplastic epithelium, goblet cell depletion, and mononuclear cell infiltration into the epithelium (Fig. 1g). However, Ad-DRA mice displayed significantly less histopathologic evidence of colitis, a lower histologic score $(16.56 \pm 1.62 N=9$ vs. $31.38 \pm 1.34 N=$ $8, P<0.01$, Fig. 1d, g) and a relatively intact intestinal epithelium. The intestinal permeability was determined using Evans Blue (EB). The amount of EB that permeated into the intestinal wall in the DSS-induced group was $387.5 \pm 12.09 \mu \mathrm{g} / \mathrm{g}(N=6)$, which was markedly higher than that of the Ad-DRA group $(128.7 \pm 10.96 \mu \mathrm{g} / \mathrm{g} N=6$, $P<0.01$, Fig. 1e), suggesting mild epithelial barrier dysfunction in the Ad-DRA group. However, there was a statistically significant difference between the normal group $(89.47 \pm 6.0 \mu \mathrm{g} / \mathrm{g}, \quad N=6)$ and the Ad-DRA group $(P<0.05)$. TEM was used to visualize the ultrastructure of the epithelial barrier and TJs. Figure $1 \mathrm{~h}$ shows an intact TJ structure in the normal control mice. In the Ad-0 control group, discontinuous zones were easy to find, and there was less electron-dense material present between adjoining cells near the brush border, indicating a disruption of normal TJ morphology. The Ad-DRA group exhibited intensive and regular microvilli and ameliorated TJs.

We also analyzed TJ protein expression (Fig. 1i). Ad-0 control mice displayed markedly reduced DRA, ZO-1, occludin and claudin 5 expression but enhanced claudin 2 expression in response to DSS treatment. Compared with Ad-0 control mice, total occludin and ZO-1 protein levels were reduced to a significantly smaller extent in Ad-DRA mice but were not fully restored. Ad-DRA treatment efficiently prevented the effects of DSS on TJ protein levels.

We also examined whether Ad-DRA treatment was associated with modifications in pro- and anti-inflammatory cytokine production (Fig. 1j). Ad-0-treated animals displayed significantly elevated levels of TNF- $\alpha$, IL- 6 and KC (all $P<0.05$ compared with the normal control group). IL10 secretion in the normal control and Ad-0 control groups was low with no significant difference between these two groups $(P>0.05)$. However, Ad-DRA treatment dramatically suppressed proinflammatory cytokine (TNF- $\alpha$, IL-6 and $\mathrm{KC})$ levels while increasing IL-10 expression $(P<0.05$ 

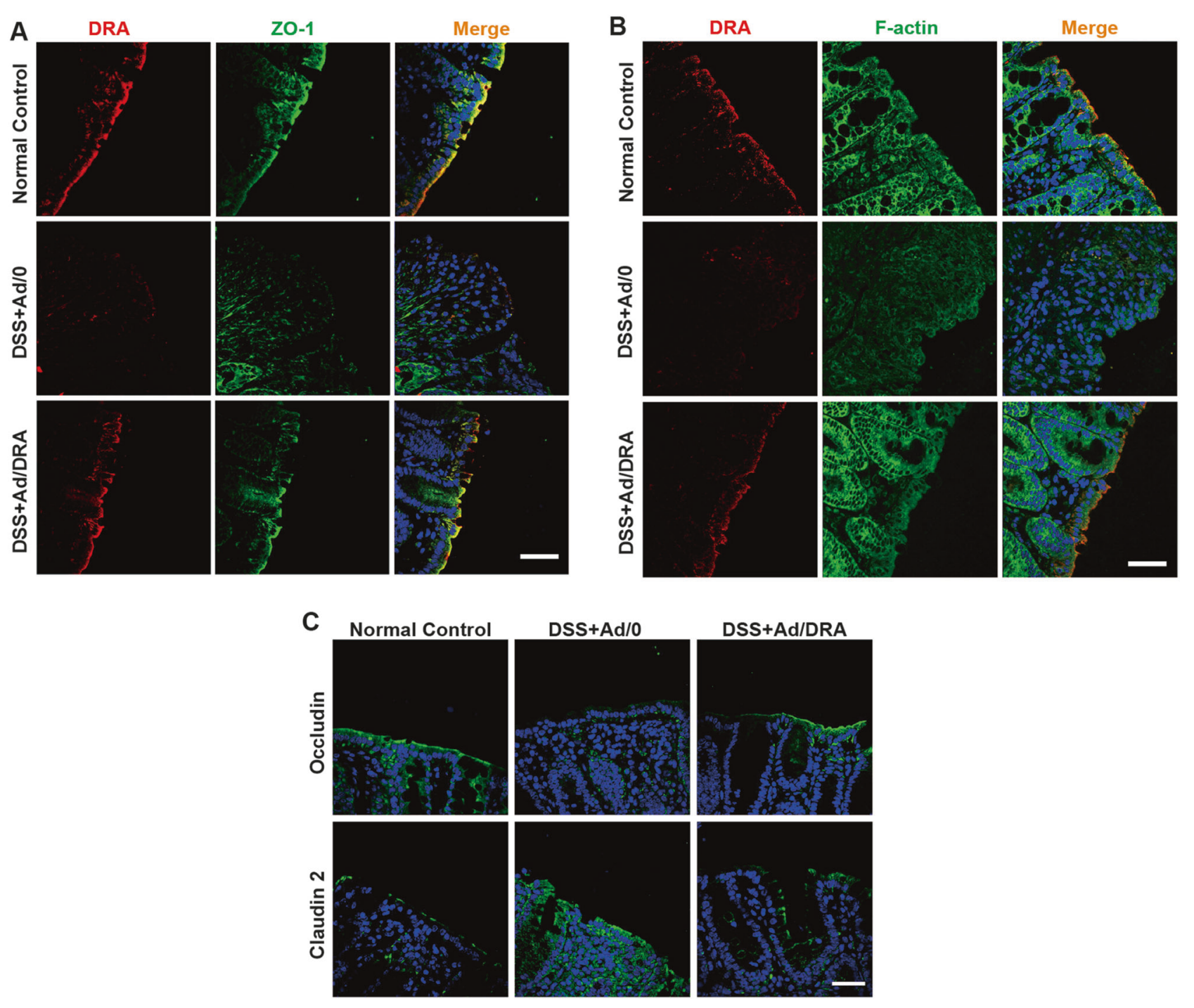

Fig. 5 Delivery of Ad-DRA stabilizes TJ proteins. a Confocal immunofluorescence of DRA (red) and ZO-1 (green) in each group. Nuclei were stained with DAPI (blue). Scale bar $=50 \mu \mathrm{m}$. DRA colocalized with the tight junction protein ZO-1. b Confocal immunofluorescence of DRA (red) and F-actin (green) in each group. Scale bar $=50 \mu \mathrm{m}$. DRA was concentrated near the apical margin of the

compared with the Ad-0 control group). No differences in TNF- $\alpha$, IL- 6 and KC levels were observed between the AdDRA and normal control groups $(P>0.05)$, indicating the inhibition of inflammation in the Ad-DRA group.

To analyze the localization of colonic DRA, IF staining and confocal laser scanning microscopy were performed. Previous work clearly showed that DRA is strongly expressed in the distal colon [27]. Therefore, we chose the distal colon as a representative site. In normal mice, DRA was expressed on the cell surface and colocalized with the TJ protein ZO-1 (Fig. 5a). Intense DRA staining was typically concentrated near the apical margin of the surface beyond F-actin in the normal colon (Fig. 5b). In Ad-0 surface, beyond F-actin, in the normal colon. In the Ad-DRA group, DRA was typically present in epithelial cells but not colocalized with F-actin. c Immunofluorescence staining of distal colon tissues with occludin and claudin 2 antibodies. Scale bar $=50 \mu \mathrm{m}$

control mice, DSS caused substantial depletion of the normal epithelium structure, along with inflammatory cell infiltration, leading to the complete loss of ZO-1 and F-actin staining. Delivery of Ad-DRA led to increased expression of DRA in the epithelium and colocalization with ZO-1 compared with Ad-0 control group. The damage caused by DSS was still observed in Ad-DRA mice but was much less severe than that in Ad- 0 control mice, as reflected by F-actin remodeling at the epithelial cell surface. Importantly, DRA was typically observed in epithelial cells without colocalization with F-actin, and F-actin was concentrated beneath DRA in the Ad-DRA group. We also observed single staining of occludin and claudin 2. In normal mice, occludin 
A

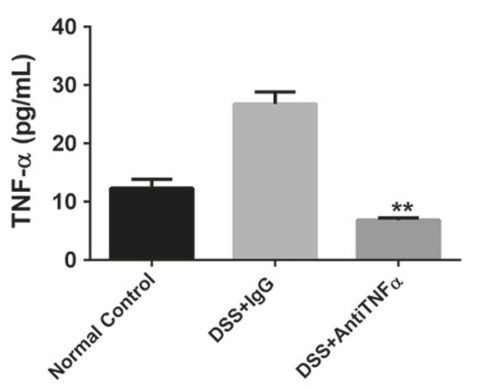

C
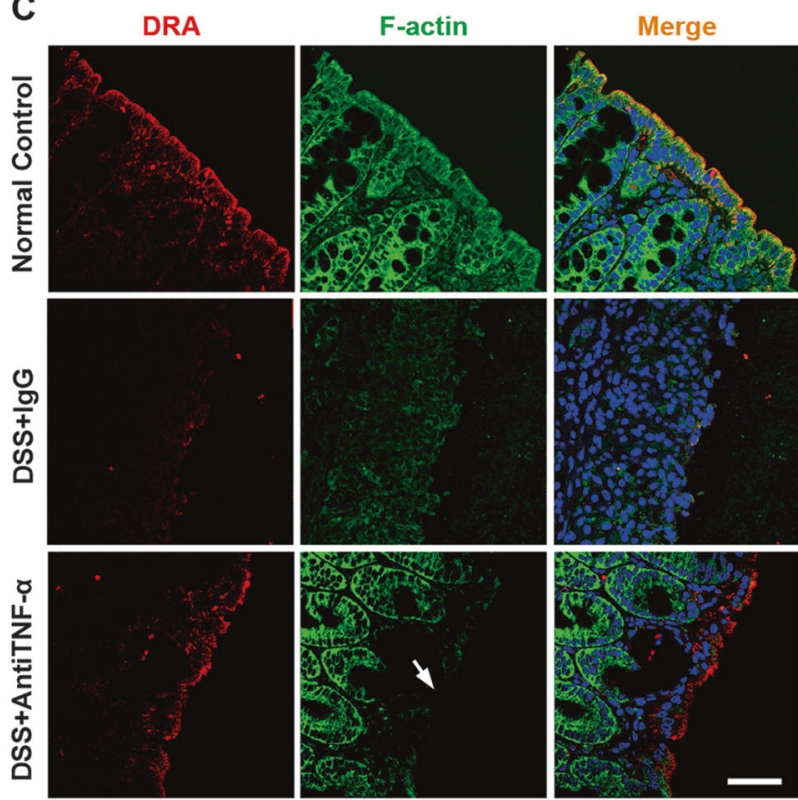

Fig. 6 DRA is retained in anti-TNF $\alpha$-treated colitis mice and associated with changes in TJ expression and F-actin. a Modulation of TNF- $\alpha$ levels by the TNF- $\alpha$ mAb. The TNF- $\alpha$ concentration (in pg/ $\mathrm{mL})$ data are presented as the mean $\pm \mathrm{SE}$ of 6 mice per group $(* * P<$ 0.01 , one-way ANOVA). Intraperitoneal injection of the TNF- $\alpha$ mAb on days 4 and 6 significantly neutralized TNF- $\alpha$ in serum. b Double immunofluorescence staining for DRA (red) and ZO-1 (green) in colonic tissue sections from control mice (top), DSS-treated IgG control mice (middle) and DSS- and TNF- $\alpha$ mAb-treated mice (bottom). DRA predominantly localized at the apical region of injured colonic epithelial cells, and colocalization with retained ZO-1 in TNF$\alpha$ mAb-treated mice. Scale bar $=20 \mu \mathrm{m}$. c Immunofluorescence for

was appropriately localized at the colonic epithelial apical cell-cell contacts, which is consistent with ZO-1 localization. In the normal control group, almost no claudin 2 signal was detected. Ad-DRA treatment recovered occludin staining and reduced the elevated claudin 2 levels (Fig. 5c).

\section{Anti-TNF-a treatment prevents the loss of DRA in DSS-induced colitis}

Administration of a TNF- $\alpha$ mAb was effective at blocking TNF- $\alpha$ in serum at day 10 (Fig. 6a, $P<0.01$ ). Consistent with the previous observations $[18,28], \quad \mathrm{TNF}-\alpha$
B DRA
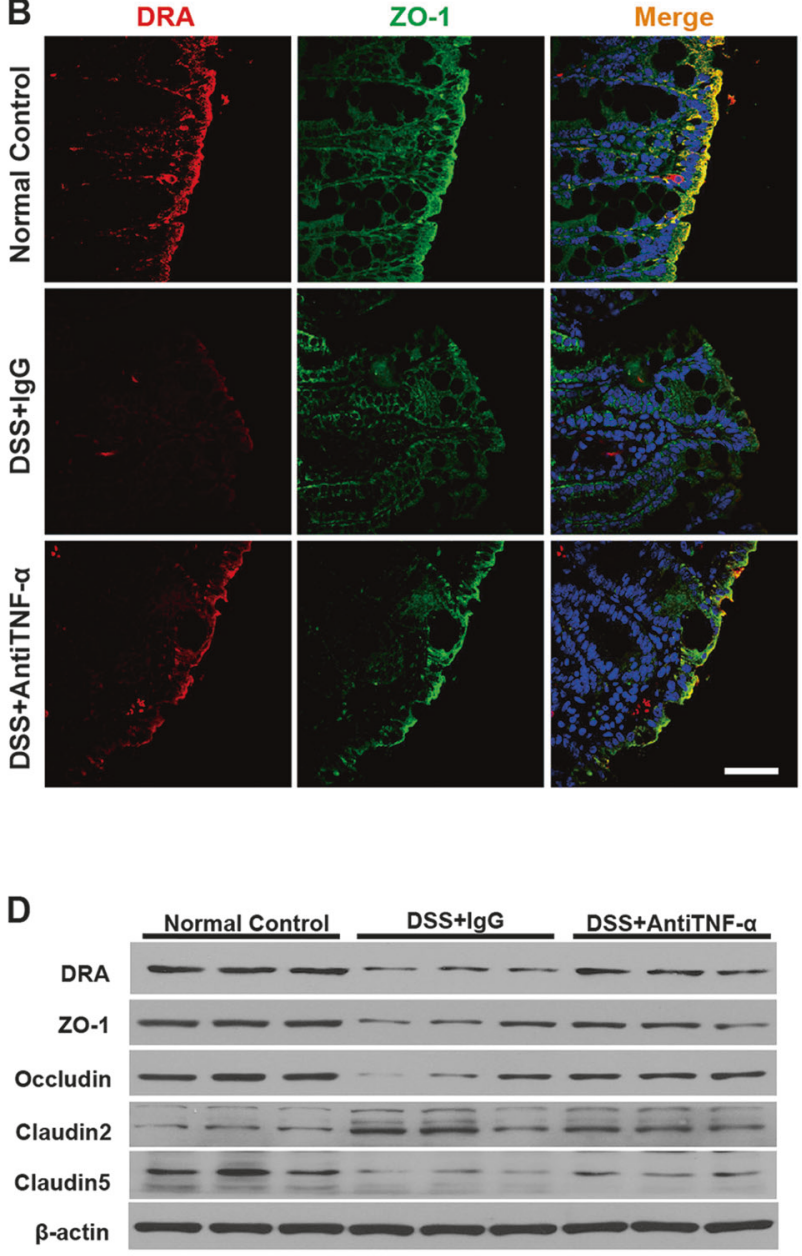

DRA and F-actin (green) in colonic tissue sections from control mice (top), DSS-treated IgG control mice (middle) and DSS- and TNF- $\alpha$ $\mathrm{mAb}$-treated mice (bottom). Scale bar $=20 \mu \mathrm{m}$. DSS treatment caused a dramatic reorganization of F-actin in TNF- $\alpha \mathrm{mAb}$-treated mice and weakened the fluorescence intensity in some areas, as indicated by the arrow. d Consistent with the immunofluorescence results, anti-TNF $\alpha$ treatment resulted in higher DRA expression. The expression of TJ proteins, including occludin and ZO-1, was significantly higher compared with the $\mathrm{IgG}$ control group, whereas claudin 2 was not affected. Claudin 5 expression obviously decreased in both DSS-treated groups

neutralization resulted in less severe acute colitis compared with IgG1 isotype treatment (Supplementary Fig. 2). TNF- $\alpha$ $\mathrm{mAb}$ treatment has been associated with more favorable outcomes, including a lower DAI, less weight loss and a longer colon, compared with IgG control. In addition, the increase in intestinal permeability was significantly smaller in TNF- $\alpha$ mAb-treated mice than in IgG control-treated mice, as shown in the EB assay and electron microscopy, suggesting an improvement in colon function and the effectiveness of the TNF- $\alpha$ mAb treatment.

The previously described pattern of DRA localization with ZO-1 and F-actin in normal mice was observed. 
A

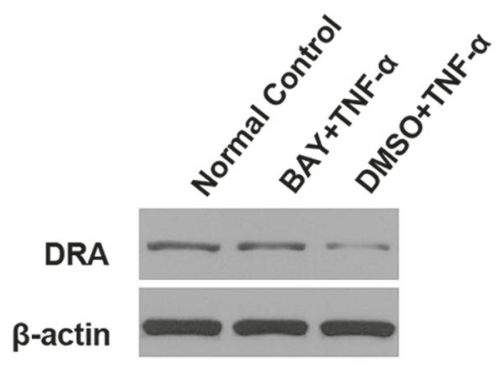

C

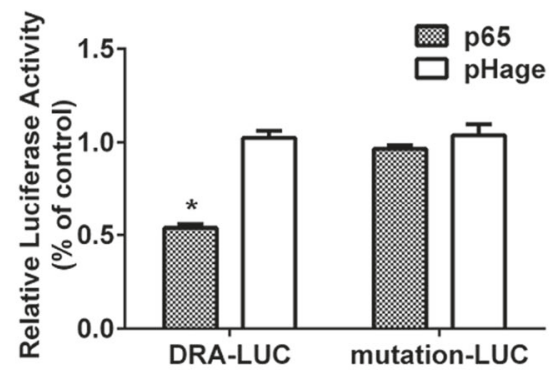

B
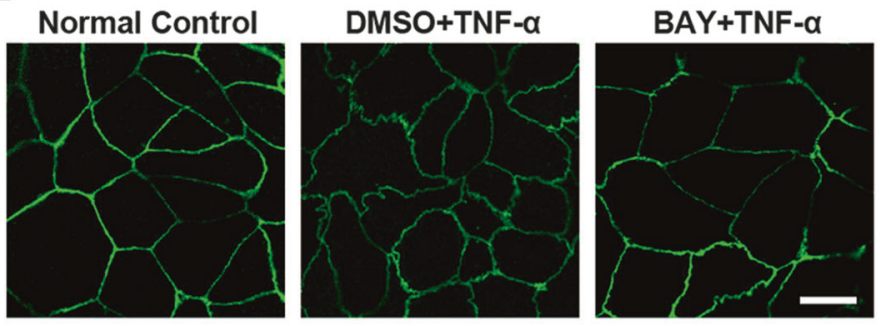

D

NF-kB WT: GGGGAATATTCTTT
NF-KB MUT: GCAGAATATTGTT

TNF- $\alpha$ PBS

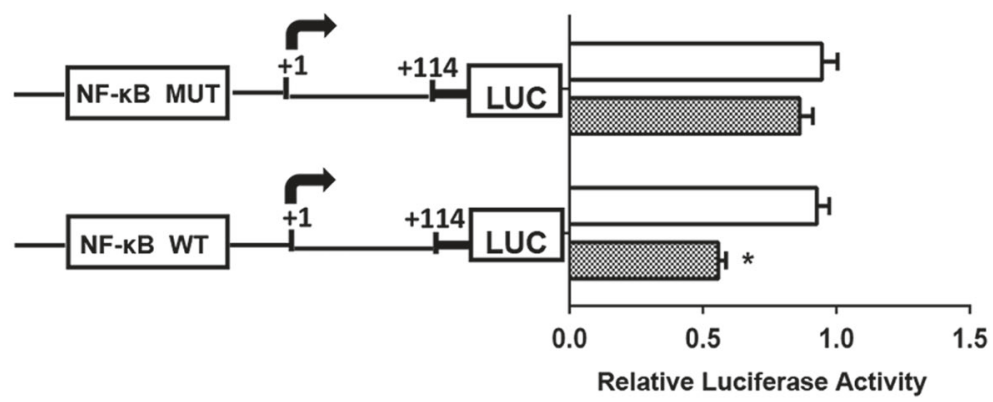

Fig. 7 TNF- $\alpha$ inhibits DRA expression via the NF-кB pathway. The results are presented as the $\%$ of control transfected cells, and the data were obtained from 3 separate experiments. $* P<0.05$ compared with control. a Caco-2BBe cells were pretreated with BAY-11-7082 for 30 min and then treated with TNF- $\alpha(100 \mathrm{ng} / \mathrm{mL})$ for $6 \mathrm{~h}$. BAY-11-7082 treatment prevented the TNF- $\alpha$-induced decrease in DRA. b Immunofluorescence detection of ZO-1. In the DMSO control group, ZO-1 showed morphological changes. BAY-11-7082 treatment preserved normal ZO-1 morphology, which implied the stabilization of TJs. c Identification of the role of $\mathrm{NF}-\mathrm{KB} / \mathrm{p} 65$ in DRA promoter regulation.

However, DSS- and IgG control-treated mice displayed a dramatic reduction in DRA staining intensity, which led to a complete loss of colocalization with ZO-1 (Fig. 6b) and Factin (Fig. 6c) at the apical membrane. In contrast, TNF- $\alpha$ mAb-treated mice showed a less severe decrease in DRA staining intensity with less epithelial damage. Notably, DRA predominantly localized at the apical region of injured colonic epithelial cells, and colocalization with ZO-1 was retained. DSS treatment caused a dramatic reorganization of F-actin in TNF- $\alpha$ mAb-treated mice and resulted in some regions of weakened fluorescence intensity (arrow, Fig. 6c). DRA was still present and accumulated in the terminal web region but did not colocalize with diffuse F-actin. Although TNF- $\alpha$ mAb treatment prevented the loss of DRA, the levels of DRA remained diminished, as manifested by discontinuities in some areas and a reduction in membrane staining intensity.

To extend our observations of altered TJs, we analyzed the expression of TJ proteins by western blot. Along with changes in IF staining, TNF- $\alpha$ mAb treatment prevented the
Caco-2BBe cells were transiently transfected with the p65/control vector (pHage), wild-type or mutant NF-кB DRA-LUC promoter construct and the pRL-TK vector. Data were normalized to pRL-TK activity to correct for transfection efficiency. $\mathbf{d}$ The NF-кB-binding site is responsible for DRA promoter regulation in response to TNF- $\alpha$. Caco-2BBe cells were transfected with the WT or mutant NF-кB DRA-LUC promoter construct and the pRL-TK vector and then treated with $100 \mathrm{ng} / \mathrm{mL}$ of TNF- $\alpha$ for $6 \mathrm{~h}$ in media

loss of DRA and the colonic TJ proteins ZO-1 and occludin. Claudin 2 levels were increased significantly in $\mathrm{IgG}$ control mice but were not much lower in TNF- $\alpha$ mAb-treated mice. Much lower levels of claudin 5 were observed in both the TNF- $\alpha$ mAb and IgG control groups (Fig. 6d).

\section{NF-KB mediates the TNF- $a-$ Induced decrease in DRA}

Activation of the TNF- $\alpha / \mathrm{NF}-\mathrm{\kappa B}$ pathway has been shown to contribute to barrier dysfunction in IBD [29]. As shown in Figs. 7a and 7b, BAY-11-7082 treatment attenuated the reduction in DRA upon TNF- $\alpha$ stimulation and preserved normal ZO-1 morphology compared with the DMSO control group. These results suggest that TNF- $\alpha / \mathrm{NF}-\kappa \mathrm{B}$ signaling is likely involved in regulating DRA expression. We therefore investigated the molecular mechanisms underlying the transcriptional modulation of SLC26A3 gene expression by TNF- $\alpha$. The databases identified one common highly conserved consensus sequence in the DRA promoter region $(-1137$ to $-1128 \mathrm{bp})$, which was also mentioned by 


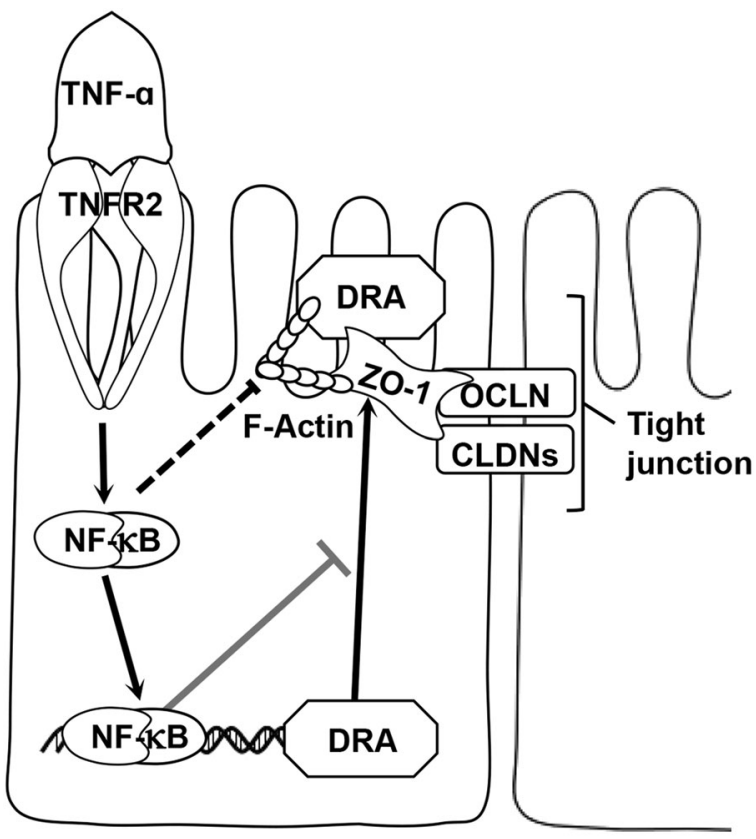

Fig. 8 Proposed model of the potential mechanisms of TNF- $\alpha$ mediated TJ dysfunction via the inhibition of DRA gene expression in human intestinal epithelial cells

Saksena and colleagues [30]. To confirm the role of this NF-кB-binding site in inhibiting DRA promoter activity, we mutated this site in a DRA promoter luciferase reporter construct (DRA-LUC; Fig. 7d). In WT DRA-LUC Caco$2 \mathrm{BBe}$ cells transfected with the p65 plasmid, a significant reduction in luciferase activity was observed compared with transfection with the vector control (pHage) $(0.54 \pm 0.02$ vs. $1.03 \pm 0.04, P<0.05)$. On the other hand, luciferase activity was unchanged in the MUT DRA-LUC Caco-2BBe cell line $(0.97 \pm 0.02$ vs. $1.04 \pm 0.06, P>0.05)$, suggesting that this NF- $\mathrm{KB}$-binding site plays an important role in regulating DRA expression (Fig. 7c). Furthermore, we examined the effects of TNF- $\alpha$ on DRA promoter activity. Caco-2BBe cells were transfected with WT or MUT DRA-LUC and then incubated with or without TNF- $\alpha(100 \mathrm{ng} / \mathrm{mL})$ for $6 \mathrm{~h}$. As shown in Fig. 7d, TNF- $\alpha$ treatment resulted in a marked decrease in WT DRA-LUC activity $(0.56 \pm 0.03$ vs. $0.93 \pm$ $0.05, P<0.05)$ but no significant difference in MUT DRALUC activity $(0.87 \pm 0.05$ vs. $0.95 \pm 0.06, P>0.05)$. All results were obtained from three independent experiments, and the data suggest that NF- $\mathrm{KB}$ is involved in modulating the DRA promoter in response to TNF- $\alpha$.

\section{Discussion}

Many studies have proposed therapeutic approaches for IBD such as probiotics and peptides targeting impaired TJs and disordered gut barrier function [31-34]. TJs consist of transmembrane proteins, such as $\mathrm{ZO}-1$ and occludin, and the perijunctional actin cytoskeleton, which together mediate adhesive functions. These proteins provide the general basis for selective paracellular permeability in epithelial cells [35-38]. Moreover, ZO-1 was reported to be directly associated with claudins and occluding [39]. Here we observed the colocalization of ZO-1 and DRA as representative of TJs.

In the present study, we investigated the influence of DRA on the epithelial barrier and TJ protein expression, and the mechanisms of regulation in a mouse model of acute colitis and in Caco-2BBe cells. Caco-2BBe cells exhibit a well-developed brush border, and they have been shown to be polarized and structured similar to human enterocytes in vivo [40]. In polarized Caco-2BBe cells, DRA colocalized with ZO-1 directly affected the expression of $\mathrm{TJ}$ proteins. These colocalization and co-IP results suggest that DRA and ZO-1 potentially interact, which lays a foundation for further study. In addition, we found that prolonged treatment with high-dose TNF- $\alpha$ disturbed ZO-1, occludin and F-actin expression, which correlated with DRA downregulation and internalization from TJ complexes. Moreover, DRA overexpression prevented the TNF- $\alpha$-mediated alteration of $\mathrm{TJ}$ proteins and other negative effects to a certain extent compared with the normal control, suggesting that overexpressing DRA strengthened the epithelial barrier antagonized by high-dose TNF- $\alpha$. In contrast, DRA KD was associated with the disruption of TJ proteins and an impaired epithelial barrier. These results indicate that DRA ensures intestinal barrier function, in part through stabilizing TJs. Herein, we delineate a novel function of DRA in TJ maintenance.

To investigate the possible mechanism by which TNF- $\alpha$ decreases DRA expression, we used a dual-luciferase reporter system to assess the effect of mutating the predicted NF-кB-binding site in the DRA promoter region. The abnormal NF-кB activation regulated by TNF receptor signaling was taken as evidence that this pathway contributes to the pathogenic functions of TNF- $\alpha$ in IBD [41]. Moreover, an NF-кB inhibitor showed therapeutic effects against colitis in mice [42]. Our research indicated that this pathway actively regulates DRA, and sequence analysis of the DRA promoter revealed a potential NF-кB-binding site. We observed that mutation $(-1137$ to $-1128 \mathrm{bp})$ of the DRA promoter region disrupted the TNF- $\alpha$-induced reduction in DRA-LUC activity compared with the WT control, suggesting that the TNF- $\alpha$-induced inhibition of DRA promoter activity is mediated by NF-кB. Our finding is consistent with a recent study by Kumar et al, who also showed that TNF- $\alpha$ decreases DRA expression by activating NF-кB [43]. There are indirect signaling pathways between TNF- $\alpha$ and DRA. For example, the intestinal 
transcription factor $\mathrm{CDX} 2$ has been reported as a downstream target of TNF- $\alpha$ and a direct transcriptional activator of the DRA promoter [44]. Numerous intracellular signaling molecules, including protein kinases and phosphatases, are involved in regulating TJs [45]. Although we have shown that DRA maintains intestinal epithelial barrier integrity via TJs, AJs were not investigated in this study. Therefore, we cannot exclude the possibility that DRA has other targets in addition to TJs, and further research on this topic is needed. A schematic representation of the mechanism of TJ regulation through DRA downregulation by $\mathrm{TNF}-\alpha / \mathrm{NF}-\kappa \mathrm{B}$ is shown in Fig. 8.

To determine the biological effects of DRA, we also conducted in vivo experiments. Previous work has shown that normal mature intestinal epithelial cells are extremely resistant to transduction by adenovirus, whereas tissue from IBD patients is transduced significantly more readily than normal tissue [46]. Therefore, Ad-DRA was administered on days 1, 3, and 5 following the initial DSS treatment. Consistent with the in vitro data, delivery of Ad-DRA ameliorated disease activity and reduced the loss of TJ proteins, which protect the epithelial barrier from harmful substances in the colonic lumen. The experimental group showed a significant reduction in both outcomes and ultrastructural pathology. Notably, ultrastructure observations by electron microscopy showed that the intercellular junction was sealed in Ad-DRA mice, thus providing a structural basis for the blockade of pathogenic factors in the gut. Consistent with the in vitro results, Ad-DRA treatment preserved DRA expression in colonic epithelial cells, thereby stabilizing the TJ structure and maintaining the epithelial barrier. Distinctive cytokine profiles have been identified with significant correlations with disease duration and activity [47]. Delivery of Ad-DRA reduced proinflammatory cytokine levels, perhaps due to the enhanced epithelial barrier function, which could prevent harmful factors from breaking through the epithelial barrier.

We also observed that TNF- $\alpha$ neutralization in mice with colitis mitigated the loss of DRA and alleviated the epithelial barrier damage (Supplementary Figure 2). Intraperitoneal administration of the TNF- $\alpha$ mAb improved colitis in mice, as has been reported in other studies [18, 29, 30]. We observed DRA recovery in response to TNF- $\alpha \mathrm{mAb}$ treatment even before the recovery of F-actin, which suggested that DRA recovers before restoration of the epithelium. In other words, DRA is an intermediate event between TNF- $\alpha$ mAb treatment and restoration of the epithelial barrier. These results may highlight the protective role of DRA in the colitis model.

Therefore, both in vivo and in vitro experiments showed that the prevention of DRA downregulation is protective against TNF- $\alpha$-induced barrier loss. Because these two animal studies were not performed at the same time, and the time points and intervention methods were different, the two therapeutic effects could not be compared. However, we found that DRA overexpression in mice significantly reduced diarrhea and blood in stool (Supplementary Fig. 1). Moreover, the water content of stool (\%) at the end of the observation period (the 10th day) was significantly lower in mice with DRA overexpression than in TNF $\alpha$ mAb-treated mice $(54.02 \pm 1.03$ vs. $64.89 \pm 1.53 \%, P<0.01)$. In TNF- $\alpha$ mAb-treated mice, thickening and significant edema of the intestinal wall were observed by visual inspection and electron microscopy. In contrast, we observed shaped feces in most tissue samples from Ad-DRA-treated mice, which may have been due to the fluid-electrolyte absorption function of DRA.

Because there is no effective treatment for IBD, and up to $40 \%$ of patients exhibit primary nonresponsiveness to anti-TNF therapy or complications [48, 49], new therapeutic strategies are needed. The risk of undesired effects may be lower with other specific strategies that directly target the epithelium. However, the field of gene therapy is currently focused on protective immune responses and cytokines, and still presents enormous challenges regarding biosafety, target cell specificity, suitable vector systems, and delivery methods. Nevertheless, DRA has demonstrable biological effects in animal experiments, which may provide a basis for future human studies.

In conclusion, our in vitro and in vivo data show that DRA plays a role in protecting the epithelial barrier in intestinal inflammation. The present study shows that, in addition to participating in fluid-electrolyte absorption and acid/base balance in the gut, as well as mucus layer barrier formation, DRA is involved in the epithelial barrier and in preventing the progression of inflammation. Our study characterized a new mechanistic pathway in IBD, in which DRA interacts with the TNF- $\alpha / N F-\kappa B$ pathway by targeting TJ proteins. The multiple roles of DRA may inform novel therapeutic strategies for the management of IBD in the future.

Acknowledgements We thank Prof. Ursula Seidler (Hannover Medical School, Germany) for the generous gift of the human colon epithelial Caco-2BBe cell line. This work was supported by the National Natural Science Foundation of China (No. 81400593 to Yu Qin and No. 81572419 to Tian Dean).

\section{Compliance with ethical standards}

Conflict of interest The authors declare that they have no competing interests.

\section{References}

1. Molodecky NA, Soon IS, Rabi DM, et al. Increasing incidence and prevalence of the inflammatory bowel diseases with time, based on systematic review. Gastroenterology. 2012;142:46-54. 
2. Priyamvada S, Gomes R, Gill RK, et al. Mechanisms underlying dysregulation of electrolyte absorption in inflammatory bowel disease-associated diarrhea. Inflamm Bowel Dis. 2015;21:2926-35.

3. Diamond JM. Twenty-first Bowditch lecture. The epithelial junction: bridge, gate, and fence. Physiologist. 1977;20:10-8.

4. van Meer G, Simons K. The function of tight junctions in maintaining differences in lipid composition between the apical and the basolateral cell surface domains of MDCK cells. EMBO J. 1986;5:1455-64.

5. Bruewer M, Samarin S, Nusrat A. Inflammatory bowel disease and the apical junctional complex. Ann $\mathrm{N}$ Y Acad Sci. 2006;1072:242-52.

6. Turner JR. Intestinal mucosal barrier function in health and disease. Nat Rev Immunol. 2009;9:799-809.

7. Pastorelli L, De Salvo C, Mercado JR, et al. Central role of the gut epithelial barrier in the pathogenesis of chronic intestinal inflammation: lessons learned from animal models and human genetics. Front Immunol. 2013;4:280.

8. Xia W, Yu Q, Riederer B, et al. The distinct roles of anion transporters Slc26a3 (DRA) and Slc26a6 (PAT-1) in fluid and electrolyte absorption in the murine small intestine. Pflug Arch. 2014;466:1541-56.

9. Ishiguro $\mathrm{H} . \mathrm{HCO}_{3}{ }^{-}$secretion by SLC26A3 and mucosal defence in the colon. Acta Physiol. 2014;211:17-9.

10. Farkas K, Yeruva S, Rakonczay Z Jr., et al. New therapeutic targets in ulcerative colitis: the importance of ion transporters in the human colon. Inflamm Bowel Dis. 2011;17:884-98.

11. Yang H, Jiang W, Furth EE, et al. Intestinal inflammation reduces expression of DRA, a transporter responsible for congenital chloride diarrhea. Am J Physiol. 1998;275:G1445-53.

12. Xiao F, Juric M, Li J, et al. Loss of downregulated in adenoma (DRA) impairs mucosal $\mathrm{HCO}_{3}{ }^{-}$secretion in murine ileocolonic inflammation. Inflamm Bowel Dis. 2012;18:101-11.

13. Walker NM, Simpson JE, Hoover EE, et al. Functional activity of Pat-1 (Slc26a6) $\mathrm{Cl}^{-} / \mathrm{HCO}_{3}{ }^{-}$exchange in the lower villus epithelium of murine duodenum. Acta Physiol. 2011;201:21-31.

14. Xiao F, Yu Q, Li J, et al. Slc26a3 deficiency is associated with loss of colonic $\mathrm{HCO}_{3}{ }^{-}$secretion, absence of a firm mucus layer and barrier impairment in mice. Acta Physiol. 2014;211:161-75.

15. Bhutia YD, Babu E, Ramachandran S, et al. SLC transporters as a novel class of tumour suppressors: identity, function and molecular mechanisms. Biochem J. 2016;473:1113-24.

16. Juric M, Xiao F, Amasheh $\mathrm{S}$, et al. Increased epithelial permeability is the primary cause for bicarbonate loss in inflamed murine colon. Inflamm Bowel Dis. 2013;19:904-11.

17. Yeruva S, Chodisetti G, Luo M, et al. Evidence for a causal link between adaptor protein PDZK1 downregulation and $\mathrm{Na}^{+} / \mathrm{H}^{+}$ exchanger NHE3 dysfunction in human and murine colitis. Pflug Arch. 2015;467:1795-807.

18. Lopetuso LR, Petito V, Cufino V, et al. Locally injected Infliximab ameliorates murine DSS colitis: differences in serum and intestinal levels of drug between healthy and colitic mice. Dig Liver Dis. 2013;45:1017-21.

19. Wirtz S, Neurath MF. Gene transfer approaches for the treatment of inflammatory bowel disease. Gene Ther. 2003;10:854-60.

20. Williams KL, Fuller CR, Dieleman LA, et al. Enhanced survival and mucosal repair after dextran sodium sulfate-induced colitis in transgenic mice that overexpress growth hormone. Gastroenterology. 2001;120:925-37.

21. Sutherland LR, Martin F, Greer S, et al. 5-Aminosalicylic acid enema in the treatment of distal ulcerative colitis, proctosigmoiditis, and proctitis. Gastroenterology. 1987;92:1894-8.

22. Halpert G, Eitan T, Voronov E, et al. Multifunctional activity of a small tellurium redox immunomodulator compound, AS101, on dextran sodium sulfate-induced murine colitis. J Biol Chem. 2014;289:17215-27.
23. Liu XC, Mei Q, Xu JM, et al. Balsalazine decreases intestinal mucosal permeability of dextran sulfate sodium-induced colitis in mice. Acta Pharmacol Sin. 2009;30:987-93.

24. Kitajima S, Takuma S, Morimoto M. Changes in colonic mucosal permeability in mouse colitis induced with dextran sulfate sodium. Exp Anim. 1999;48:137-43.

25. Arrieta MC, Madsen K, Doyle J, et al. Reducing small intestinal permeability attenuates colitis in the IL10 gene-deficient mouse. Gut. 2009;58:41-8.

26. Barmeyer C, Schulzke JD, Fromm M. Claudin-related intestinal diseases. Semin Cell Dev Biol. 2015;42:30-8.

27. Talbot C, Lytle C. Segregation of $\mathrm{Na}^{+} / \mathrm{H}^{+}$exchanger-3 and $\mathrm{Cl}^{-} /$ $\mathrm{HCO}_{3}{ }^{-}$exchanger SLC26A3 (DRA) in rodent cecum and colon. Am J Physiol Gastrointest Liver Physiol. 2010;299:G358-67.

28. Xiao YT, Yan WH, Cao Y, et al. Neutralization of IL-6 and TNF$\alpha$ ameliorates intestinal permeability in DSS-induced colitis. Cytokine. 2016;83:189-92.

29. Fischer A, Gluth M, Pape UF, et al. Adalimumab prevents barrier dysfunction and antagonizes distinct effects of TNF- $\alpha$ on tight junction proteins and signaling pathways in intestinal epithelial cells. Am J Physiol Gastrointest Liver Physiol. 2013;304:G970-9.

30. Saksena S, Singla A, Goyal S, et al. Mechanisms of transcriptional modulation of the human anion exchanger SLC26A3 gene expression by IFN- $\gamma$. Am J Physiol Gastrointest Liver Physiol. 2010;298:G159-66.

31. Chen Y, Zhang HS, Fong GH, et al. PHD3 stabilizes the tight junction protein occludin and protects intestinal epithelial barrier function. J Biol Chem. 2015;290:20580-9.

32. Dai $\mathrm{C}$, Zhao DH, Jiang M. VSL\#3 probiotics regulate the intestinal epithelial barrier in vivo and in vitro via the p38 and ERK signaling pathways. Int J Mol Med. 2012;29:202-8.

33. Zhang Y, Brenner M, Yang WL, et al. Recombinant human MFGE8 ameliorates colon damage in DSS- and TNBS-induced colitis in mice. Lab Invest. 2015;95:480-90.

34. Saijo H, Tatsumi N, Arihiro S, et al. Microangiopathy triggers, and inducible nitric oxide synthase exacerbates dextran sulfate sodium-induced colitis. Lab Invest. 2015;95:728-48.

35. Tsukita S, Katsuno T, Yamazaki Y, et al. Roles of ZO-1 and ZO-2 in establishment of the belt-like adherens and tight junctions with paracellular permselective barrier function. Ann N Y Acad Sci. 2009;1165:44-52.

36. McCarthy KM, Skare IB, Stankewich MC, et al. Occludin is a functional component of the tight junction. J Cell Sci. 1996;109:2287-98.

37. Watson AJ. Claudins and barrier dysfunction in intestinal inflammation: cause or consequence? Gut. 2015;64:1501-2.

38. Zeissig S, Burgel N, Gunzel D, et al. Changes in expression and distribution of claudin 2, 5 and 8 lead to discontinuous tight junctions and barrier dysfunction in active Crohn's disease. Gut. 2007;56:61-72.

39. Odenwald MA, Choi W, Buckley A, et al. ZO-1 interactions with F-actin and occludin direct epithelial polarization and single lumen specification in 3D culture. J Cell Sci. 2017;130:243-59.

40. Sambuy Y, De Angelis I, Ranaldi G, et al. The Caco-2 cell line as a model of the intestinal barrier: influence of cell and culturerelated factors on Caco-2 cell functional characteristics. Cell Biol Toxicol. 2005;21:1-26.

41. Pasparakis M. Role of NF-кB in epithelial biology. Immunol Rev. 2012;246:346-58

42. Funakoshi T, Yamashita K, Ichikawa N, et al. A novel NF-кB inhibitor, dehydroxymethylepoxyquinomicin, ameliorates inflammatory colonic injury in mice. J Crohns Colitis. 2012;6:215-25.

43. Kumar A, Chatterjee I, Gujral T et al. Activation of NF-кB by tumor necrosis factor in intestinal epithelial cells and mouse intestinal epithelia reduces expression of the chloride transporter SLC26A3. Gastroenterology. 2017;153:1338-50. 
44. Chatterjee I, Kumar A, Castilla-Madrigal RM, et al. CDX2 upregulates SLC26A3 gene expression in intestinal epithelial cells. Am J Physiol Gastrointest Liver Physiol. 2017;313(3):G256-64.

45. Wang F, Schwarz BT, Graham WV, et al. IFN- $\gamma$-induced TNFR2 expression is required for TNF-dependent intestinal epithelial barrier dysfunction. Gastroenterology. 2006;131:1153-63.

46. Schmiedlin-Ren P, Kesisoglou F, Mapili JA, et al. Increased transduction of human intestinal epithelial cells by adenoviral vectors in inflammatory bowel disease. Inflamm Bowel Dis. 2005;11:464-72.
47. Alex P, Zachos NC, Nguyen T, et al. Distinct cytokine patterns identified from multiplex profiles of murine DSS- and TNBSinduced colitis. Inflamm Bowel Dis. 2009;15:341-352.

48. West NR, Hegazy AN, Owens BMJ, et al. Oncostatin M drives intestinal inflammation and predicts response to tumor necrosis factor-neutralizing therapy in patients with inflammatory bowel disease. Nat Med. 2017;23:579-89.

49. Guerra I, Bermejo F. Management of inflammatory bowel disease in poor responders to infliximab. Clin Exp Gastroenterol. 2014;7:359-67. 